\title{
China's National Research Project on Wireless Sensor Networks
}

\author{
Lionel M. Ni \\ Department of Computer Science and Engineering \\ The Hong Kong University of Science and Technology \\ Clear Water Bay, Kowloon, Hong Kong \\ ni@cse.ust.hk
}

\begin{abstract}
This talk will give an overview of the 5-year National Basic Research Program of China (also known as 973 Program) on Wireless Sensor Networks launched in September 2006 sponsored by Ministry of Science and Technology. This national research project involving researchers from many major universities in China and Hong Kong with an aim to tackle fundamental research issues rose in three major application domains: coal mine surveillance, water pollution monitoring, and traffic monitoring and control. The distinctive feature of the project is that it will present a systematic study of wireless sensor networks, from node platform development, core protocol design and system solution development to critical problems. This talk will address the research challenges, current progress, and future plan.
\end{abstract}

